\title{
LA SOCIALIZACIÓN DIFERENCIAL EMOCIONAL DE GÉNERO COMO FACTOR PREDICTOR DEL CARÁCTER
}

\section{EMOTIONAL DIFFERENTIAL SOCIALIZATION OF GENDER AS A PREDICTOR OF THE CHARACTER}

\author{
Iratxe Suberviola Ovejas. Universidad de La Rioja-España \\ ratxe.suberviola@unirioja.es
}

\begin{abstract}
Resumen Este artículo se enclava en la actual corriente que considera que el análisis de las competencias emocionales por cuestión de género necesita matización, puesto que en ocasiones los resultados aportados están referidos al "sexo" y no al "género". En las siguientes páginas se realiza un análisis de diferentes investigaciones sobre competencias emocionales y la variable "sexo"|"género" estableciendo tres categorías: competencias emocionales y "sexo"; competencias emocionales y "género"; competencias emocionales y su relación con la socialización diferencial emocional de género. Tras el análisis se apunta que teniendo en cuenta el vínculo entre las habilidades emocionales y la formación del carácter, el género sería un factor predictor de este.
\end{abstract}

Palabras claves Inteligencia Emocional; Competencias Emocionales; Género; Carácter.

Abstract This article follows the current that considers that the analysis of emotional competences and gender needs clarification because sometimes the results are referred to "sex" and not "gender". This document is an analysis of different research about emotional competences and the variable "sex"l"gender". There are three categories: emotional competences and "sex"; emotional competencies and "gender" and; emotional competencies and its relationship with the emotinal differential socialization of gender. The analysis indicates, keep it in mind the bond between emotional habilities an character training, the gender would be a predictor of this.

Keys words Emotional Inteligence; Emotional Competences; Gender; Character.

\section{Introducción}

Las emociones, además de facilitar la adaptación del individuo al entorno físico, posibilita la acomodación a su entorno social, por lo tanto, teniendo en cuenta que las personas somos seres bio-socio-emocionales y que las emociones son influenciadas e influyentes en los aspectos biológicos, psicológicos y sociales, las emociones van a constituirse como elementos fundamentales en la configuración de la personalidad de cada individuo. A la hora de hablar de personalidad se deben tener en cuenta dos términos que sustentan dicho concepto, como son: el temperamento y el carácter. (Montaño, Palacios y Gantiva, 2009). 
El temperamento es un fenómeno naturalmente emocional, es decir, que se presenta a causa de factores genéticos o hereditarios, pues los individuos reaccionan de manera rápida e intensa ante la estimulación ambiental y por tanto su estado puede fluctuar de acuerdo a las exigencias del medio. Desde las primeras etapas de la evolución humana, las necesidades de adaptación del hombre primitivo y de los animales superiores impulsaron el desarrollo de los instintos básicos, como la huida, defensa y reproducción, donde las emociones básicas juegan un papel importante. (Allport, 1975)

Respecto al segundo término que compone el concepto de personalidad se encuentra el carácter que es entendido como la combinación de sentimientos, valores y emociones que un individuo va adquiriendo a lo largo de su desarrollo a través de la interacción, condiciones y circunstancias externas, además difiere en cada individuo de acuerdo con su forma o punto de vista de interpretar la realidad humana, debido a que cada persona se ve influenciada por diferentes factores que ocurren a su alrededor. Por tanto, como lo plantea Lluís (2002), el carácter controla, modifica, corrige y autorregula la actividad de los individuos, a fin de poder dar respuestas satisfactorias a las exigencias del medio. En definitiva, el carácter se configura en relación a los estereotipos de género que marcan cada sociedad y lo que el contexto espera de cada individuo por el hecho de ser hombre o mujer.

Es importante destacar que las emociones se construyen principalmente en la interacción social, aunque son interiorizadas como patrón emocional personal. Desde esta perspectiva, debemos plantearnos que el carácter $y$, por lo tanto la personalidad que desarrolla cada individuo, está condicionada en parte por la denominada socialización de género y concretamente, por la socialización diferencial emocional de género, puesto que limita las posibilidades de expresión de sentimientos al determinar la expresión diferencial de los mismos en hombres y mujeres, (Rebollo, Hornillo y García, 2006) influyendo en la configuración de la personalidad, puesto que tal como se ha mencionada en párrafos anteriores, el carácter depende de las experiencias de cada individuo y está condicionado por factores e interacciones socio-culturales.

La socialización diferencial emocional de género existente entre mujeres y hombres implica la consideración social de que niños y niñas son en esencia diferentes y están llamados a desempeñar papeles y roles emocionales diferentes. Así, los agentes socializadores, como son: el sistema educativo, la familia, los medios de comunicación, el grupo de iguales, etc., tienden a asociar tradicionalmente la masculinidad con el poder, la racionalidad, aspectos de la vida social pública, la política, el trabajo profesionalidad, la regulación de las expresiones emocionales, etc., es decir, tareas productivas que responsabilizan a los varones de los aspectos materiales y no tanto con el mundo de los sentimientos. Sin embargo, las féminas son asociadas con la pasividad, la dependencia, la obediencia y aspectos de la vida privada, como el cuidado o la afectividad, en definitiva, tareas de reproducción que responsabilizan a las mujeres de los aspectos emocionales. (Rebollo y Hornillo, 2010; Suberviola, 2018). 


\section{Método}

En las siguientes páginas se realiza una revisión de diferentes investigaciones en relación a las Competencias Emocionales teniendo en cuenta la perspectiva de género. En dicho análisis se establecen tres categorías de estudios en referencia a la profundidad con la que las variables "sexo"|"género" son tratadas, tomando el "sexo" como el referente a la biología, a las diferencias físicas/biológicas entre los cuerpos de hombres y mujeres y el "genero" en palabras de la OMS, como los roles socialmente construidos, los comportamientos, actividades y atributos que una sociedad dada considera apropiados para los hombres y las mujeres.

1) Estudios sobre Competencias Emocionales en los que se incluye la variable sexo. En estas pesquisas el "sexo" es tratado como algo meramente estadístico, sin tener en cuenta la educación y la socialización previa de cada persona, es decir, sin ahondar en cuestiones de género (Gartzia, Antxeta, Balluerka y Barberá, 2012; Pérez-Pérez y Castejón, 2005).

2) Estudios sobre Competencias Emocionales en los que se incluye la variable "género". En estas investigaciones el "género" hace referencia a la construcción social de mujeres y hombres, de feminidad y masculinidad, que varía en el tiempo y el espacio y entre las culturas. Se aleja de la noción de "sexo" para señalar que la biología o la anatomía no son un sino. Abordar la dimensión del género en la investigación implica que el género se considera una variable clave analítica y explicativa en la investigación. (Ministerio de Ciencia e Innovación, 2011)

3) Estudios sobre Competencias Emocionales y su relación con la socialización diferencial emocional de género, entendida como el proceso de aprendizaje por el que se transmiten las creencias, los valores, los comportamientos dominantes de una determinada sociedad, se construyen las relaciones de género y se asignan los papeles emocionales diferenciales. (Junta de Andalucía, 2019)

\section{Estudios sobre Competencias Emocionales en los que se incluye la variable "sexo"}

En las últimas décadas se está produciendo un incremento de trabajos sobre Competencias Emocionales, su importancia y su correlación con ciertos aspectos de la personalidad. En la mayoría de ellos se realizan un análisis teniendo en cuenta el "sexo" como variable. Como anteriormente hemos apuntado, estos estudios no contemplan las representaciones culturales constituidas por ideas, perjuicios, valores, interpretaciones, normas, deberes, prohibiciones, etc., que recaen sobre hombres y mujeres (Colas y Villaciervos, 2007), sino que únicamente realizan el análisis desde un paradigma biológico.

Uno de ellos es el realizado por el equipo de Fernández-Berrocal y Extremera (2003) con alumnado universitario de los últimos cursos de psicopedagogía donde se encontró que las estudiantes obtuvieron una mayor puntuación en malestar personal que sus compañeros varones, relacionándolo con una mayor atención a sus sentimientos. Este hecho se 
correlaciona con los resultados obtenidos en secundaria donde las mujeres obtienen puntuaciones más elevada en atención a los sentimientos y, como consecuencia, mayor sintomatología ansiosa. Estos factores se han asociado a una peor salud mental comparativamente con los hombres que presentan una mayor capacidad para regular las emociones negativas (Extremera y Fernández-Berrocal, 2003).

Corroborando los datos obtenidos por los autores anteriormente citados, en una investigación llevada a cabo por Carreteo y Nolasco (2016), con 540 estudiantes del Grado de Magisterio, se concluye que el rasgo de atención emocional está significativamente más presente en las mujeres, mientras que los hombres presentan una mayor regulación emocional.

Bar-On, Brown, Kirkcaldy y Thome (2000) desarrollan una investigación en las que se examinan las dimensiones de expresividad emocional en diferentes ocupaciones utilizando el EQ-i (Bar-On Emotional Quotient Inventory) (Bar-On, 1997). Los resultados indican que no hay diferencias significativas entre sexos en Inteligencia Emocional general. Sin embargo, las mujeres presentan mejores habilidades interpersonales, mientras que los varones toleran mejor el estrés y controlan mejor los impulsos. Estos resultados coinciden con la investigación desarrollada por Reyna y Brussino (2015), donde a través de la información obtenida por medio de las familias y docentes de 627 niños/as argentinos/as entre 3 y 10 años se determina que las féminas presentan mejores habilidades sociales y mayores competencia interpersonales.

En un trabajo de Dawda y Hard (2000) desarrollado con el EQ-i no se hallaron diferencias significativas en las puntuaciones totales. Sin embargo, las mujeres puntuaron más alto que los hombres en el factor de responsabilidad social, mientras que los varones obtuvieron mayores puntuaciones en independencia y optimismo.

En la literatura científica, además de los estudios anteriormente mencionados, existen otros que afirman que las mujeres suelen ser emocionalmente más expresivas que los varones, tienen una mayor comprensión de las emociones y muestran mayor habilidad en ciertas competencias interpersonales, es decir, que interpretan mejor las expresiones de los demás y son más empáticas (Aguiano, 2003; Lafferty, 2004; Tapia y Marsh, 2006).

En un estudio desarrollado por Pérez-Pérez y Castejón (2005) en la Universidad de Alicante (España), los datos apuntan que, a pesar de no existir diferencias claras en aspectos de Inteligencia Emocional, sí se percibe una mayor autorregulación emocional en los estudios técnicos, en los cuales la población masculina es mayoritaria. Sin embargo, se apreciaron diferencias claras en la variable sexo, tanto en el conjunto de componentes de la Inteligencia Emocional, como en algunos aspectos concretos. Es de destacar la mayor atención emocional que prestan las mujeres, así como la mayor claridad, reparación y regulación emocional de los hombres.

Por otro lado, Eisler y Blalock (1991) estudiaron como la manifestación emocional de los hombres es diferente e inferior al de las mujeres. Éstas manifiestan detalles más íntimos sobre ellas y expresan más emociones, tanto positivas como negativas.

Un estudio realizado en Lima (Ugarriza, 2001) con 1.996 personas mayores de 14 años, halló que el "sexo" tiene efectos diferenciales para la mayoría de los componentes 
factoriales. En este sentido, se demostró que los hombres tienen un mejor autoconcepto, piensan que solucionan mejor los problemas, toleran mejor el estrés y tiene un mayor control de los impulsos. Las mujeres, sin embargo, obtienen más ventajas en sus relaciones interpersonales, mejor empatía y una mayor responsabilidad social.

Sánchez, Retana y Carrasco (2008) realizaron un estudio con personas comprendidas entre los 18 y los 27 años sobre la comprensión de las emociones, como una de las principales competencias de la Inteligencia Emocional. Las autoras encontraron que las mujeres tienen mayor capacidad para comprender los contextos ideales de la emoción relacionadas con la tristeza, y las emociones complejas y contradictorias.

En un análisis llevado a cabo con 338 trabajadores por Gartzia, et al. (2012) se concluye que la aceptación de rasgos de expresividad por parte de las mujeres podría explicar sus mayores puntuaciones en Inteligencia Emocional. En este mismo sentido, en un estudio realizado por Martínez-Marín y Martínez-Martínez (2016) en la Universidad de Murcia, se determinó que las mujeres se identifican con rasgos de identidad expresivos en mayor medida que los hombres y esto influye en la mejora de las competencias emocionales tales como la sensibilidad, la socialización y la atención a la necesidad de los demás.

Molero, Ortega y Moreno en 2010, midieron los componentes de la Inteligencia Emocional a través del TMMS-24 (Trait Meta-Experience Scale) (Fernández-Berrocal, Extremera y Ramos, 2004) con una muestra de 704 estudiantes de las titulaciones de magisterio con especialidades de infantil, primaria, música, lengua extranjera y educación física. Los resultados mostraron significatividad en las variaciones de "sexo" en la escala de Percepción y atención a los sentimientos a favor de las mujeres y la escala de Regulación o reparación de las emocionales, a favor de los hombres.

En un estudio similar al anterior, con muestra de estudiantes universitarios de Grado de Infantil y Primaria de la Universidad de la Rioja (España), en la que se medía la Inteligencia Emocional y sus componentes a través de la herramienta de autoinforme anteriormente mencionada TMMS-24. Los resultados obtenidos apuntan la existencia de diferencias significativas entre hombres y mujeres en la competencia de atención/percepción emocional. Por el contrario, los datos señalan una mayor regulación emocional a favor de los hombres (Suberviola y Santiago, 2011).

En un estudio reciente, diseñado por Molero y Pantoja en 2015, utilizando la misma herramienta que en los estudios anteriormente mencionado, con una muestra de 27 alumnos de grado de primaria (magisterio) en prácticas en los centros escolares y 43 docentes en ejercicio, comprobaron que las mujeres puntuaban más alto que los hombres en las tres subescalas, es decir, percepción, comprensión y regulación emocional.

\section{Estudios sobre Competencias Emocionales en los que se incluye la variable "género"}

Partiendo de la veracidad de los estudios anteriormente expuestos, podríamos pensar que las Competencias Emocionales están condicionadas por las diferencias de "sexo"I"género". Sin embargo, nos debemos plantear que la totalidad de estas investigaciones se han llevado a cabo en personas con un bagaje emocional tanto en el ámbito formal como no formal. Esto nos debería llevar a cuestionarnos si las Competencias 
Emocionales están determinadas por el "sexo" o lo están por el "género". Que en un momento determinado del ciclo vital las mujeres y los hombres obtengamos puntuaciones diferentes en ciertas Competencias Emocionales no significa que en los primeros años de vida hayan existido dicha variación.

De acuerdo con lo que se acaba de señalar, son varios los estudios que apuntan que es necesario ahondar en la línea de investigación que aborda el estudio del género, y no únicamente el "sexo" para determinar cuáles son las causas de las diferencias observadas entre los hombres y las mujeres en el eficaz manejo de las Competencias Emocionales. (Joseph y Newman, 2010; Salovey, 2006).

En este sentido, algunas pesquisas muestran como las mujeres presentan mayor patología depresiva que los hombres, sin embargo, esta sintomatología no es una constante en la mujer desde su nacimiento, sino que surge en la adolescencia. Los niños preadolescentes manifiestan desordenes depresivos y ansiógenos en una proporción similar que sus compañeras (Altemus, Sarvay y Epperson, 2014). No obstante, a partir de la adolescencia los estudios señalan que comienzan las diferencias de género apreciándose en las chicas una mayor rumiación, lo que provoca un mayor nivel de ansiedad y aumento de la depresión. (Resurreción, Ruiz-Aranda y Salguero-Noguera, 2016).

En otro estudio desarrollado en el mismo sentido, llevado a cabo en Colombia por Oyuela y Pardo (2003) sobre las diferencias de género en el reconocimiento de las expresiones faciales emocionales, se obtienen datos que muestran una tendencia de las mujeres a reconocer con mayor facilidad las expresiones de alegría, mientras que los hombres parecen reconocer mejor las de ira. Estos autores argumentan que estas diferencias pueden estar relacionadas con el vínculo que se establece entre la madre y el neonato donde la sonrisa es uno de los factores centrales. Sin embargo, los hombres reconocerían con mayor facilidad las expresiones de ira puesto que, etiológicamente, de su reconocimiento dependía la supervivencia de la especie.

Esto supone una interesante conclusión puesto que apunta que las diferencias en competencias emocionales no son apreciables en los primeros momentos de vida, es decir, no tendría una base genética. En este sentido, Brody y Hall (2000) determinaron que, aunque en principio no existe diferencia manifiesta en la expresividad facial de chicos y chicas, a lo largo de la escuela primaria los primeros se van volviendo menos expresivos, mientras que las niñas aumentan su expresividad.

En un estudio realizado por Reyna y Brussino en 2015, se determina como los niños y niñas de 3 años muestran un comportamiento social inadecuado en la misma medida. Sin embargo, ya desde los 5 años, las féminas presentan niveles más altos de habilidades sociales y más bajos de problemas de conducta, mientras que los varones de esta misma edad estuvieron en niveles intermedios.

Los resultados de un estudio llevado a cabo por Reyes y Mora (2007) con 24 niños y 24 niñas con edades comprendidas entre 54 y 60 meses de edad de la ciudad de Bogotá, donde se analizaba la regulación emocional a través de acciones conflictivas con títeres, no arrojaron diferencias significativas entre niños y niñas, corroborando un estudio previo llevado a cabo por Adams et al. (1995) que afirmaba que las diferencias emocionales de expresión y regulación solo son observables a partir de los 70 meses de edad. 
Una de las conclusiones más interesantes y recientes al respecto es la realizada por Martínez-Marín y Martínez-Martínez (2016) quienes afirman que las diferencias que se dan en algunos componentes de la Inteligencia Emocional entre hombres y mujeres tienen que ver en mayor medida con el "género" y no tanto con el "sexo", y que es algo más cultural que biológico. De este modo, la identidad de género femenino de las madres se relaciona con la misma identidad de género que las hijas, al igual que los padres femeninos tienen hijos con esa misma identidad de género. En este sentido, tal como se muestra en un estudio realizado por Sánchez, Retana y Carrasco en 2008, los resultados confirman que la identidad de género ejerce influencia sobre la Inteligencia Emocional evaluada tanto a través de medidas de autopercepción (TMMS) como de habilidad (MSCEIT). Concretamente, los resultados corroboran que, a diferencia de otros tipos de identidad de género, la identidad andrógina se relaciona con niveles superiores de Inteligencia Emocional en todas las dimensiones analizadas.

\section{Estudios sobre Competencias Emocionales y su relación con la socialización de género}

En el anterior apartado se han mencionado algunas investigaciones sobre competencias emocionales bajo la perspectiva de género. En ellas se apunta que la construcción social del género y los estereotipos marcados para hombres y mujeres podrían estar bajo la diferencia en la adquisición de Competencias Emocionales. Algunos estudios analizados en los que se toma la edad como variable, darían consistencia a esta hipótesis apuntando que las variaciones en habilidades emocionales son más tenues en los primeros años de vida, debido a una menor exposición a una socialización diferencial emocional de género, tal y como concluyen Carretero y Nolasco (2016) y Garaigordobil (2013), afirmando que desde la niñez las niñas están en contacto, en mayor medida que los niños, con las Competencias Emocionales, al igual que consideran que la socialización y educación de las niñas se desarrolla con un mayor contacto con las emocionales y sus matices.

Si buscamos el origen de las diferencias debemos retrotraernos hasta edades tempranas donde se forjan muchas de las competencias emocionales. Diversos estudios muestran las diferencias emocionales a los que niños y niñas se ven expuestos por los agentes socioemocionales.

Un ejemplo que visibiliza la diferente relación entre los/as progenitores/as y sus descendientes, según el "sexo" de los segundos, es la utilización de los cuentos como herramienta de educación informal. Debemos tener presente que en los cuentos los términos que aparecen con mayor frecuencia son los emocionales, seguidos de los cognitivos. (Rabazo y Moreno, 2006) Diferentes investigaciones muestran que cuando los padres y madres narran cuentos a sus hijos/as pequeños/as, suelen utilizar palabras más cargadas emocionalmente con las niñas, además de usar más el discurso emocional con éstas al discutir sobre eventos que connotan tristeza. La diada padre/madre-hija sitúa la experiencia emocional en un contexto más interpersonal que la diada padre/madre-hijo (Fivush, Brotman, Buchner y Goodmen, 2000).

Siguiendo con la socialización de los progenitores, en relación a la expresión emocional Scharfe (2000) encontró cómo la expresión maternal y el sexo del descendiente estaban asociados con diferencias individuales en la expresividad del niño o la niña y que estas 
diferencias eran estables en el tiempo. Las madres mostraban mayor expresividad con las chicas que con los chicos y, a los tres años, las madres informaban de más afecto positivo en las chicas y más afecto negativo en los chicos. Sin embargo, otros estudios como el realizado por Dunn en 1990 muestra que en las familias en las que la madre y el niño varón tienen conversaciones sobre estados emocionales, los niños suelen tener una consciencia e interés emocional mayor.

En este sentido, Sánchez, Fernández-Berrocal, Montañes y Latorre (2008) encontraron que los padres tienden a hablar más sobre emociones con sus hijas que con los hijos. Además, las madres usan más términos emocionales que los padres cuando juegan con sus descendientes. Otro aspecto a determinar en esta línea es que los progenitores utilizan un espectro más amplio de emocionales en el caso de que el grupo de receptores esté únicamente formado por féminas, sumando a ello que son más detallistas con ellas cuando describen su estado emocional.

Otro importante elemento diferencial a destacar en la socialización de las competencias emocionales la encontramos en la instrucción sobre las técnicas de resolución de problemas por parte de las familias, dónde se encuentra una alta correlación entre dicha instrucción y las habilidades emocionales posteriores, especialmente en hombres. Esto se debe a que los padres instruyen a los niños en la resolución de problemas y reservan la instrucción para las chicas, únicamente en el caso de que se perciba un déficit importante en este aspecto. (Jones et al. 2002).

Siguiendo con la socialización diferencial de género, en un exhaustivo análisis realizado por Campbell en California en 2014, se concluyó que la manera más importante sobre el modo de que los padres brindan un trato diferente a las hijas con respecto a los chicos, es a través de actividades estereotipadas de género, en la que se incluye el tipo de juguetes que los padres compran a sus descendientes, promoviendo actividades más tranquilas y emotivas para las féminas, a través de muñecas y juegos de rol relacionados con la familia y juguetes más cinéticos, técnicos y deportivos para ellos.

Otro de los contextos más importantes para la socialización de las emociones, actitudes y comportamiento de género de los infantes de corta edad, es sin duda las escuelas, tanto en la relación con los docentes como entre iguales. Los estereotipos y prejuicios de género del profesorado se manifiestan en el aula desde tres vertientes. (Bigler, Roberson y Hamilton, 2013) La primera se aprecia en el modelo de comportamiento estereotipado de género donde las profesoras se muestran más cercanas y emotivas. La segunda, el grupo de docentes frecuentemente muestran expectativas diferentes con respecto a los alumnos que a las alumnas y también en la futura elección de estudios. La tercera es que el profesorado facilita sesgos de género a los infantes, utilizando la categoría género como un hándicap importante a la hora de distribuir al alumnado en el trabajo por grupos.

\section{Discusión / Conclusiones}

Tal y como se ha podido apreciar en la revisión realizada sobre investigaciones en referencia a la Inteligencia y Competencias Emocionales tomando en consideración las variables "sexo"|"género", se ha podido comprobar la existencia de diferencias significativas, entre hombres y mujeres, en algunas habilidades relacionadas con las emociones y la expresión y regulación de las mismas. No obstante, al ahondar más en el 
tema, se determina como dichas diferencias no se aprecian en un grado representativo en edades tempranas, sino que por el contrario se acentúan con el paso de la edad, debido a una socialización diferencial emocional de género, que es llevada a cabo a través de los principales y más tempranos agentes socio-educativos como son la educación formal y no formal por parte de las instituciones educativas y las familias. Debido a ello, se establecen roles emocionales diferentes para los diferentes géneros.

La reflexión anterior está en la línea de la investigación desarrollada por Sánchez, Fernández-Berrocal, Montañes y Latorre (2008) que estudiaron la socialización diferencial de género de las Competencias Emocionales concluyendo que las mujeres tienen un mayor índice de Inteligencia Emocional con respecto a los hombres, pero su autopercepción tiende a ser más baja y que estas diferencias de puntuaciones empiezan a vislumbrarse desde edades tempranas a consecuencia de una instrucción diferencial.

Como ya se ha mencionado en la introducción, el carácter es entendido como una combinación de sentimientos, valores y emociones que un individuo va adquiriendo a lo largo de su desarrollo a través de la interacción, condiciones y circunstancias externas. Una vez analizada la existencia de una socialización diferencial emocional de género, se podría apuntar que el carácter de las personas estaría marcado por la influencia de dicha socialización diferencial, y de ese modo, el carácter de mujeres y hombres estaría modelado por los agentes socioeducadores y la intensidad con que la lleven a la cabo.

Nuestras formas de actuar, pensar, sentir, emocionarnos y expresarlo están determinadas por las normas, valores y modelos de nuestra sociedad, por lo que, podemos decir que hay una orientación normativa que nos lleva a actuar y ser de una u otra manera. Estas normas culturales que guían la conducta son aceptadas por los individuos que forman la sociedad, es decir, si mi acción se rige por las normas culturales comunes, es aceptada por los demás, y a su vez yo acepto la de los demás. Podemos decir que hay una expectativa recíproca, una interacción social regulada por normas, que de forma más o menos consciente, está determinada por los roles de género que marcan las competencias emocionales que hombres y mujeres deben tener y que no siempre tienen que ser coincidentes.

Se debe tener en cuenta que, a través de la socialización de género las sociedades instruyen a sus niños y niñas para que absorban los comportamientos considerados apropiados para cada sexo. Durante el proceso de socialización se crea el imaginario social y su forma la identidad de género de cada persona (Gobierno de Navarra, 2017) y el comportamiento y estereotipos emocionales vinculados al mismo.

En este sentido convienen destacar que los estudios que han centrado el análisis en las diferencias de género en Inteligencia Emocional a través de escalas auto informadas contrastan con las realizadas a través de escalas de ejecución, mostrando que los hombres sobrevaloran sus habilidades emocionales. Sin embargo, las mujeres las infravaloran presentando mayor ejecución emocional que lo que ellas informan. (Brackett y Mayer, 2003; Brackett, River, et al., 2006) Por un lado, los cuestionarios o medidas de autoinforme hacen referencia a la información que el sujeto nos proporciona acerca de la percepción que tiene sobre sus propias competencias emocionales, respondiendo una serie de cuestiones clave, compuestas por enunciados verbales cortos, en los que la persona estima sus niveles en 
determinadas habilidades emocionales. Por otro lado, las medidas de ejecución o pruebas de habilidad se asemejan a las pruebas tradicionales de medición del rendimiento cognitivo o pruebas de inteligencia, donde a los sujetos se les plantea resolver determinados problemas emocionales y, posteriormente, su respuesta es comparada con criterios de puntuación predeterminados y objetivos. En consecuencia, las supuestamente diferentes competencias emocionales de hombres y mujeres, queda atenuada o diluida dependiendo del tipo de instrumento de evaluación que se utilice.

Los agentes socio-educativos deben ser conocedores del sistema de "sexo"|"género" existente preestablecido en lo que respecta a las Competencias Emocionales y preparar a las personas para responsabilizarse de sus emociones sin la presión ejercida por las "normativas sociales" establecidas para los diferentes géneros. Las instituciones socioeducativas, especialmente la escuela, deben promover situaciones que posibiliten el desarrollo de la sensibilidad y el carácter de las personas, sobre la base de que en el quehacer educativo se involucra tanto el ser físico como el mental, el afectivo y el social, en un todo globalizado. (Suberviola, 2018)

\section{Referencias bibliográficas}

Adams., Kuebli, J., Boyle, P. y Tivush, R. (1995). Gender differences in parent-child conversations about pass emotions: a longitutional investigation. Sex Roles, 33 (5), 309-323.

Aguiano, A. E. (2003). Diferencias de Género y Edad en la Inteligencia Emocional de un grupo de internautas. (Tesis doctoral). Universidad Inca Garcilaso de la Vega, Líma- Perú, Facultad de Psicología y Ciencias Sociales.

Allport, G. (1975). La personalidad: configuración y desarrollo. Barcelona: Editorial Herber

Altemus, M., Sarvay., N. y Epperson, N. (2014). Sex differences in anxiety and depression clinical perspectives. HHS Public Access, 35(3), 320-330. Extraído el 30 de mayo de 2018, de https://www.ncbi.nlm.nih.gov/pmc/articles/PMC4890708.

Bar-On, R. (1997). The Emotional Quotient inventory (EQ-I): Techical Manual. Toronto: MultiHealth Systems.

Bar-On, F., Brown, J.M., Kirkcaldy, B. y Thoma, E. (2000). Emotional expression and implications for occupational stress: an application of the Emotional Quotient Inventory (EQ-i). Personality and Individual Differences, 28, 1107-1118. Extraído el 29 de mayo de 2018, de https://www.sciencedirect.com/science/article/pii/S0191886999001609.

Bigler, R., Roberson, A. y Hamilton, V. (2013). El papel de las escuelas en la socialización temprana sobre diferentes géneros. Enciclopedia sobre el Desarrollo de la Primera Infancia. Extraído el 5 de marzo de 2019, de http://www.enciclopediainfantes.com/genero-socializacion-temprana/segun-los-expertos/el-papel-de-lasescuelas-en-la-socializacion

Brackett, M. A. y Mayer, J. D. (2003). Convergent, discriminant, and incremental validity of competing measures of emotional intelligence. Personality and Social Psychology Bulletin, 29, 1147-1158. 
Brackett M.A., Rivers S.E., Shiffman S., Lerner N. y Salovey P. (2006). Relating emotional abilities to social functioning: a comparison of self-report and performance measures of emotional intelligence. Journal of personality and social psychology, 91(4), 780-95

Brody, R.F. y Hall, J.A. (2000). Gender, emotion an expression. En M. Lewin, y J.M. HavilanJones, (Eds.), Handbbok of emotions (pp.87-95) New York: Guilford Press.

Campbell, Ph.D. (2015). La socialización de género en los niños por parte de los padres. Enciclopedia sobre el Desarrollo de la Primera Infancia. Extraído el 5 de marzo de 2019, de http://www.enciclopedia-infantes.com/genero-socializacion-temprana/segunlos-expertos/la-socializacion-de-genero-en-los-ninos-por-parte

Carretero, R. y Nolasco, A. (2016). La inteligencia emocional desde la perspectiva sexista. En F. Soler., L. Aparicio., L.O. Díaz, E. Escolano y A. Rodríguez. (Eds.), Inteligencia emocional y bienestar, (pp .445-454). Zaragoza: Universidad San Jorge.

Colas, P. y Villaciervos, P. (2007). La interiorización de los estereotipos de género en jóvenes y adolescentes. Revista de investigación educativa, 25(1), 35-58.

Dawda, D. y Hart, S. (2000). Sassing emotional intelligence: reliability and validity of the Bar-On Emotional Quotient Inventory (EQ-i) in university students. Personality and Individual Differences, 28(4), 797-812.

Dunn, J. (1990). La comprensión de los sentimientos: las primeras etapas. En J. Bruner y H. Haste (Eds), La elaboración del sentido. La construcción del mundo por el niño. Barcelona:Paidós

Eisler, R.M. y Blalock, J.A. (1991). Masculine gender role stress: Scale development and component factors in the appraisal of stressful situation. Clinical Psychology Review, $11,45-60$.

Extremera, N. y Fernández-Berrocal, P. (2003). La inteligencia, claridad de las relaciones interpersonales y empatía en estudiantes universitarios. Clínica y Salud, 15(2), 117137. Extraído el 9 de mayo de 2018, de http://www.redalyc.org/html/1806/180617822001.

Fernández-Berrocal, P. y Extremera, N. (2003). ¿En qué piensan las mujeres para tener un peor ajuste emocional? Encuentros en Psicología social, 1, 255-259. Extraído el 27 de mayo de 2018 de, http://www.academia.edu/4695348/Fern\%C3\%A1ndezBerrocal_P._y_Extremera_N._2003_.En_qu\%C3\%A9_piensan_las_mujeres_para_t ener un peor ajuste emocional Encuentros en Psicolog\%C $3 \% A D a$ Social 1255 $\underline{-259}$.

Fernández-Berrocal, P., Extremera, N. y Ramos, N. (2004). Validity and reliability of the Spanish modified version of the Trait Meta-Mood Scale. Psychological Report, 94, 751-755.

Fivush, R., Brotman, M., Burckner, J. P. y Goodman, S. H. (2000). Gender differences in parent-child emotion narratives. Sex Roles, 42(3), 233-253.

Garaigordobil, M. (2013). Sexism and alexithymia: Correlations and differences as a function of gender, age, and educational level. Anales de psicología, 29(2), 368-377.

Gartzia, L., Aritzeta, A., Balluerka., M.N. y Barberá, E (2012). Inteligencia emocional y género: más allá de las diferencias sexuales. Anales de psicología, 28(2), 567-575. 
Extraído el 29 de mayo de 2018, de http://revistas.um.es/analesps/article/viewFile/analesps.28.2.124111/132861.

Gobierno de Navarra. (2017). El plan coeducación 2017-2021 para los centros de las comunidades educativas de Navarra. Extraído el 8 de marzo de 2019, de https://www.educacion.navarra.es/documents/27590/1286586/Plan+de+Coeducaci\% C $3 \%$ B3n+2017-2021.pdf/5e36ddde-4bf8-ofgc-fae6-ae1462df46e2

Instituto Andaluz De La Mujer (2015). Informe sobre la campaña de juegos y juguetes 2015. Extraído el 10 de marzo de 2019, de https://www.observatoriodelainfancia.es/oia/esp/documentos ficha. $a s p x ? i d=4447$

Jones, S., Eisenberg, N., Fabes, R. y MacKinnon, D.P. (2002). Parent's reactions to elenmentary school children's negative emotion: Reations to social an emotional functioning al school. Merrill-Palmer Quarterly, 48, 133-159.

Joseph, D.L.y Newman, D.A. (2010). Emotional Intelligence: An Integra-tive Meta-Analysis and Cascading Model. Journal of Applied Psychology, 95(1), 54-78

Junta de Andalucia. Unidad de igualdad de género. (2019). Conceptos claves para comprender e incorporar el enfoque de género en la salud. Extraído el 30 de febrero de 2019, https://www.juntadeandalucia.es/institutodelamujer/ugen/modulos/Salud/socializaci on.html

Lafferte, J. (2004). The relationships between gender, empathy, an aggressive behaviors among early adolescents. Dissertation Abstracts International Section B: the Scicences and Engineering, 64(12), 6377-6389.

Lluis-Font, J.M. (2002). Personalidad. Esbozo de una teoría integradora. Psicothema, 4(1), 693-701.

Martínez-Marín, M. y Martínez, C. (2016). Relación entre la inteligencia emocional y el género. En F. Soler., L. Aparicio. L., L.O. Díaz., E., Escolano y A. Rodríguez. (Eds.), Inteligencia emocional y bienestar, (pp. 622-636). Zaragoza: Universidad San Jorge.

Mayer, J.D. y Salovey, P. (1997). What is emotional intelligence? En P. Salovey, y D.J. Sluyter, (Eds.), Emotional development and emotional intelligence, (pp.3-34). New York: Basic Books.

Ministerio de Ciencia e Innovación. (2011). Manual. El género en la investigación. Comunidad Europea: Yellow window management consultant.

Molero, D., Ortega, F. y Moreno, Mo. R. (2010). Diferencias en la adquisición de competencias emocionales en función del género. Revista electronica de Investigación y docencia, 3, 165-172.

Molero, D. y Pantoja, A. (2015). Diferencias de género en inteligencia emocional percibida en docents de infantile y primaria. Asociación Interuniversitaria de Investigación Pedagógica, 1, 368-376.

Montaño, M., Palacios, J. y Gantiva, C. (2009). Teoría de la personalidad. Un análisis histórico del concepto y su medicion. Psicología. Avances de la disciplina, 3(2), 81-107. 
Oyuela, R. y Pardo, C.F. (2003). Diferencias de género en el reconocimiento de expresiones faciales emocionales. Universitas Psychologica, 2(2), 151-168. Extraído el 29 de mayo de 2018, de http://www.redalyc.org/pdf/647/64720206.pdf.

Pérez-Pérez, N. y Castejón, J.L. (2005). Diferencias en el perfil de Inteligencia Emocional en estudiantes universitarios de distinta titulación. Psicología social y problemas sociales, 1, 197-204.

Rabazo, M.J. y Moreno, J.M. (2006). Estilo interactivo durante la lectura de cuentos infantiles de contenido engañoso. Electronic Journal Of Research in Educational Psychology, 4(3), 493-512.

Rebollo, M.A., Hornillo, I. y García, R. (2003). El estudio educativo de las emociones: Una aproximación sociocultural. Revista electrónica Teoría de la Educación: Educación y Cultura en la sociedad de la información, 7(2), 32-41.

Rebollo, M.A. y Hornillo, I. (2010). Perspectiva emocional en la construcción de la identidad en contextos educativos discursos y conflictos emocionales. Revista de educación,353, 235-263. Extraído el 1 de junio de 2018, de http://www.revistaeducacion.mec.es/re353/re353_og.pdf

Resurrección, D., Ruiz-Aranda, D. y Salguero-Noguera, J.M. (2016). Depresión y adolescencia: un análisis de los factores implicados. En F. Soler., L. Aparicio., L. O. Díaz., E. Escolano y A. Rodríguez. (Eds.), Inteligencia emocional y bienestar, (pp. $722-$ 742). Zaragoza: Universidad San Jorge.

Reyes, M.E. y Mora, C.M. (2007). Regulación emocional de la ira y diferencias de género en la edad preescolar. Psicología, Cultura y sociedad, 8, 87-110.

Reyna, C. y Brussino, S. (2015). Diferencias de edad y género en comportamiento social, temperamento y regulación emocional en niños argentinos. Acta Colombiana de Psicología, 18(2), 51-64. Extraído el 30 de mayo de 2018, de http://www.scielo.org.co/pdf/acp/v18n2/v18n2a05.pdf.

Salovey, P. (2006). Epilogue: The Agenda for Future Research. En V. Druskat, F. Sala y G. Mount (Ed.), Linking El and Performance at Work - Current Research Evidence with Individuals and Groups (pp. 267-272). Nueva York: Psychology Press.

Sánchez, M.T., Fernández-Berrocal, P., Montañes, J. y Latorre, J.M. (2008). ¿Es la inteligencia emocional una cuestión de género? Socialización de las competencias emocionales en hombres y en mujeres y sus implicaciones. Electronic Journal of Research in Educational psychology, 6 (2), 455-475.

Sánchez, R., Retana, B. E. y Carrasco, E. (2008). Evaluación psicológica del entendimiento emocional: Diferencias y similitudes entre hombres y mujeres. Revista Iberoamericana de Diagnóstico y Evaluación Psicológica, 26 (2),193-216.

Scharfe, E. (2000). Development of Emotional Expression, Understanding, and Regulationin Infants and Young Children. En R. Bar-On y J. Parker. (eds.), The Handbood of Emotional Intelligence, (pp. San Francisco: Jossey-Bass.

Suberviola, I. y Santiago, R. (2011). ¿Somos o nos hacemos emocionalmente diferentes? La variable género en la evaluación de las competencias emocionales en alumnado de la Universidad de la Rioja. III congreso Investigación y género, logros y retos. (pp. 19121924), Sevilla: Universidad de Sevilla. 
Suberviola, I. (2018). Autopercepción del profesorado de los Programas de Currículo Adaptado sobre la formación y designación docente de estas aulas. Investigación en la escuela, 94, 63-78.

Ugarriza, N. (2001). La evaluación de la inteligencia emocional a través del inventario de BarOn (I-CE) en una muestra de Lima Metropolitana. Persona, 4, 129-160.

Tapia, M. y Marsh, G.E. (2006). The effects of sex and grade point average on emotional intelligence, Psicothema, 18(supl), 108-111.

Cómo referenciar este artículo/How to reference this article:

Suberviola Oveja, I. (2020). La socialización diferencial emocional de género como factor predictor del carácter. iQUAL. Revista de Género e Igualdad, 3, 80-93, doi: 10.6018/iqual.369611

Suberviola Oveja, I. (2020). La socialización diferencial emocional de género como factor predictor del carácter.. [Emotional differential socialization of gender as a predictor of the character]. iQUAL. Revista de Género e Igualdad, 3, 80-93, doi: 10.6018/iqual.369611 